\title{
BMJ Global Health Closing the gap on institutional delivery in northern India: a case study of how integrated machine learning approaches can enable precision public health
}

Vincent S Huang, ${ }^{1}$ Kasey Morris, ${ }^{1}$ Mokshada Jain, ${ }^{1}$ Banadakoppa Manjappa Ramesh, ${ }^{2}$ Hannah Kemp, ${ }^{1}$ James Blanchard, ${ }^{2}$ Shajy Isac, ${ }^{2,3}$ Bidyut Sarkar, ${ }^{2,4}$ Vikas Gothalwal, ${ }^{2,4}$ Vasanthakumar Namasivayam, ${ }^{2}$ Pankaj Kumar, ${ }^{5}$ Sema K Sgaier (1) ${ }^{1,6,7}$

To cite: Huang VS, Morris K, Jain $\mathrm{M}$, et al. Closing the gap on institutional delivery in northern India: a case study of how integrated machine learning approaches can enable precision public health. BMJ Global Health 2020;5:e002340. doi:10.1136/ bmjgh-2020-002340

Handling editor Sanni Yaya

- Additional material is published online only. To view, please visit the journal online (http://dx.doi.org/10.1136/ bmjgh-2020-002340).

VSH and KM contributed equally.

VSH and KM are joint first authors.

Received 24 January 2020 Revised 12 August 2020 Accepted 18 August 2020

\section{Check for updates}

(c) Author(s) (or their employer(s)) 2020. Re-use permitted under CC BY-NC. No commercial re-use. See rights and permissions. Published by BMJ.

For numbered affiliations see end of article.

\section{Correspondence to}

Dr Sema K Sgaier; semasgaier@surgofoundation. org

\section{ABSTRACT}

Introduction Meeting ambitious global health goals with limited resources requires a precision public health (PxPH) approach. Here we describe how integrating data collection optimisation, traditional analytics and causal artificial intelligence/machine learning (ML) can be used in a use case for increasing hospital deliveries of newborns in Uttar Pradesh, India.

Methods Using a systematic behavioural framework we designed a large-scale survey on perceptual, interpersonal and structural drivers of women's behaviour around childbirth $(n=5613)$. Multivariate logistic regression identified factors associated with institutional delivery (ID). Causal ML determined the cause-and-effect ordering of these factors. Variance decomposition was used to parse sources of variation in delivery location, and a supervised learning algorithm was used to distinguish population subgroups.

Results Among the factors found associated with ID, the causal model showed that having a delivery plan $(\mathrm{OR}=6.1$, $95 \% \mathrm{Cl} 6.0$ to 6.3 ), believing the hospital is safer than home ( $\mathrm{OR}=5.4,95 \% \mathrm{Cl} 5.1$ to 5.6$)$ and awareness of financial incentives were direct causes of ID (OR=3.4, 95\% $\mathrm{Cl} 3.3$ to 3.5). Distance to the hospital, borrowing delivery money and the primary decision-maker were not causal. Individual-level factors contributed $69 \%$ of variance in delivery location. The segmentation analysis showed four distinct subgroups differentiated by ID risk perception, parity and planning.

Conclusion These findings generate a holistic picture of the drivers and barriers to ID in Uttar Pradesh and suggest distinct intervention points for different women. This demonstrates data optimised to identify key behavioural drivers, coupled with traditional and ML analytics, can help design a PxPH approach that maximise the impact of limited resources.

\section{INTRODUCTION}

Improving the health and well-being of people in low-income settings is challenging, especially with limited resources. Taking a

\section{Key questions}

What is already known?

- Effective behaviour change interventions require targeted approaches that take into account the varying perceptual, interpersonal and structural drivers of behaviour.

- Singular approaches to behavioural analysis, including predictive models, are inadequate to uncover the precise causal links behind decisions such as whether to give birth at home or in a healthcare facility.

\section{What are the new findings?}

- A holistic, multi-pronged analysis using machine learning (ML) techniques showed that for women in Uttar Pradesh state, India, having a delivery plan, believing that hospital is safer than home and awareness of financial incentives were direct causes of institutional delivery (ID). But these were not the case for all women.

- We identified distinct segments of women that differed in what drove their reasons to deliver, or not, in a facility.

- Some factors previously thought to be causative of ID, such as the distance to the hospital, were found to be only an indirect cause.

\section{What do the new findings imply?}

- A precision public health approach that draws on optimised data collection techniques and integrated ML methods can uncover the drivers of behaviour enabling targeted interventions and maximising the impact of limited resources.

precision public health $(\mathrm{PxPH})$ approachgetting the right intervention to the right person, at the right time and place-enables more targeted use of resources to bridge outcome gaps. ${ }^{1-5}$

Expanding global health data, novel and more comprehensive data sets, and advances 
in analytic methods are making a PxPH approach more feasible. ${ }^{6-10}$ In particular, significant progress has been made in the capability and accessibility of artificial intelligence (AI) methods. These enhance the ability to acquire and organise data, and to identify patterns and underlying heterogeneity in data that would not be possible with traditional methods. Machine learning (ML), perhaps the most common subset of AI, uses algorithms and statistical models to perform tasks without explicit instruction by relying on patterns in data. ${ }^{11} 12$

To date, most studies taking a $\mathrm{PxPH}$ approach have focused on one aspect of a precision approach, or a single analytic method. ${ }^{4}{ }^{13-16}$ However, designing and evaluating interventions without a thorough understanding of the underlying causes of the target behaviour, which often include risk and incentive perceptions, for example, among others, can be an inefficient use of time and resources, and a single intervention is often insufficient to drive change. ${ }^{17}$ The greatest potential for impact lies in an integrated intervention design for $\mathrm{PxPH}$ that can target multiple behavioural and structural aspects, using a range of analytic methods. Such an approach allows subgroups of people to be differentiated by the varying drivers behind their behaviours, for more precise targeting. Here, we demonstrate the feasibility of using multiple AI methodologies to inform a PxPH approach, with the example of promoting in-hospital births in Uttar Pradesh, India.

\section{Use case of Al for PxPH: institutional delivery in Uttar Pradesh, India}

Maternal and neonatal mortality remain stubbornly high in Uttar Pradesh, India's most populous state. For every 100000 live births, it is estimated that 201 women and 4100 infants die. ${ }^{18}{ }^{19}$ Getting women to deliver in hospital facilities instead of at home is key to address this public health crisis. ${ }^{20}$ The Indian government's programme providing women with a financial incentive to reduce out-of-pocket expenditure on institutional delivery (ID) succeeded in increasing the national ID rate from $43 \%$ to $83 \%$ in the 10 years since $2004 .^{21-24}$ Our current study finds that $18 \%$ of women in rural Uttar Pradesh continue to deliver at home. To persuade all women to choose ID, governments and policymakers need to better understand the remaining drivers and barriers to ID. This is the problem we aimed to address using a PxPH approach.

Standard data collection focuses on the what (ie, behaviour) instead of the why (ie, drivers of behaviour). Without why information, interventions are unlikely to result in impactful and sustainable behaviour change. ${ }^{25}$ Several relevant variables are available in surveys such as the National Family Health Survey. ${ }^{26}$ To collect data on a wider range of possible drivers of and barriers to women's behaviour before, during and after childbirth, we designed a comprehensive survey built on the CUBES (to Change behavior, Understand Barriers, Enablers, and Stages of change) framework. ${ }^{27}$ The variables collected included not only demographic information but also beliefs, perceptions, knowledge, role of influencers, structural factors and health behaviour patterns collected from women, their household members and community health workers (Accredited Social Health Activists or ASHAs) that visit these women. Using a combination of predictive models, causal ML and segmentation methods, we developed a holistic picture of the factors driving ID, identified high-value intervention targets and developed insights for intervention prioritisation and targeting.

\section{METHODS \\ Data}

Household survey

We designed surveys using the CUBES framework ${ }^{27}$ to enumerate the interpersonal, perceptual and contextual factors that may affect an individual's behaviour. For the purposes of this study, the primary sampling unit (PSU) was the ASHA's catchment area. Stratified random sampling of rural ASHA catchment areas was done across all 75 districts in Uttar Pradesh. The minimum required sample size was 1575 catchment areas, to support development of a stable statewide segmentation solution, as well as to generate valid and reliable inferential statistics on target maternal and newborn behaviours at state level. The sample included data from 600 blocks, with an oversampling of the 100 poorest-performing blocks on reproductive, maternal and child health outcomes. A census of all households in the catchment area was done to select all that met the screening criteria of having a woman (alive at the time of survey) who had given birth in the past 60 days (referred to as 'women' hereafter for simplicity).

Women, their husbands or other male head of household $(\mathrm{HOH})$, and the mother-in-law/matriarchs, as well as the ASHA, were interviewed after giving informed consent. Data were collected from 15120 household members (5968 women, 4199 male $\mathrm{HOH}$ and 4953 matriarchs) between September 2017 and January 2018. For all analyses presented in this article, data from women who had given birth was used, unless otherwise noted. The survey included behaviour during pregnancy, such as frequency and location of antenatal care (ANC) checkups, taking iron and folic acid supplements, and frequency of visits from ASHAs. It also assessed birth planning, ID barriers, opinion of services and infrastructure, risk perception, financial planning for delivery and awareness of government financial incentives. Demographic variables-age, religion, caste, household composition, income and proxies for wealth (eg, electricity in the home)-were also collected. The resulting data set enabled us to apply several analytic approaches to the question of why some women continue to deliver at home.

Community Behaviour Tracking Survey

We also applied our analysis to the Community Behaviour Tracking Survey (CBTS), an independently collected 
data set with some variables similar to those in the household survey. ${ }^{28}$ The CBTS is a periodic rolling survey covering women's demographics, ANC preparedness and ID. We used it primarily to validate the robustness of the causal model generated by the household survey, and to check the algorithm's robustness. To provide blocklevel estimates of key maternal and newborn health indicators across women who terminated pregnancy in the past 2 months, the required sample size per block was estimated based on the observed value of the behaviour/ service utilisation indicators and expected magnitude of change in the indicators between rounds. Given the large inter-district variations in indicator levels, the sample size varied by district. In the CBTS, the catchment area of a rural ASHA is considered as the PSU. In each block, a systematic random sample of the required number of PSUs was selected from a sampling frame consisting of all ASHA areas in the block. We used data from round 1 (February 2014-February 2015, $\mathrm{n}=57788$ ) women who terminated pregnancy within 60 days prior to interview in 100 blocks of 25 districts in Uttar Pradesh (see online supplemental material for full variable list).

\section{Patient and public involvement}

Those interviewed in the survey were not directly involved in its design, conduct, analysis or dissemination.

\section{Analyses}

Descriptive statistics

We examined descriptive statistics assessing possible correlates for home delivery among the subset of women who delivered at home. These variables were used to classify home deliveries as either elective (eg, preference for traditional village birth attendant; perceived as more convenient; belief that the hospital is not necessary) or non-elective (eg, the baby came too quickly; hospital too far; hospital fees were too high; ambulance no-show). Women could select more than one reason for home delivery; if at least one preference reason was given, the delivery was classified as elective. All analyses were weighted to account for the oversampling of the poorestperforming blocks. The primary outcome variable for all subsequent analyses was whether a mother delivered in a healthcare facility (public or private) or at home.

\section{Predictive model}

Predictive models indicate which variables are likely to be co-observed with the outcome variable. We constructed a predictive model using logistic regression to predict the delivery location. To determine which variables to include in the predictive model, we first identified a broad subset of variables that had a feasible relationship with ID (based on the temporal chain of events and expert knowledge of ID in Uttar Pradesh). We removed or combined correlated predictors, and removed predictors with little to no variance and those that were not assessed for the entire sample (due to skip patterns). This resulted in 41 predictors included in the predictive model (table 1).
Given the large number of predictor variables in the model, a p value of 0.01 (ie, $99 \% \mathrm{CI}$ ) was used as the threshold for statistical significance. Women who reported having a planned C-section were excluded from the analysis $(n=131)$. Missing data were removed using listwise deletion, leaving a final analytic sample of 5613 women (see online supplemental figure 1 for full analytical sample flowchart). The model was weighted to account for the oversampling of poorest-performing blocks.

\section{Causal ML}

Causal models indicate variables in whose absence outcome variables are unlikely to be observed. We used causal Bayesian networks (BN) for causal ML, recognising that multiple intertwined pathways can give rise to a given outcome. ${ }^{29}{ }^{30}$ Causal $\mathrm{BN}$ are probabilistic graphical models that leverage the conditional dependencies underlying a set of variables to extract causation patterns, under the assumption that all potential causes are measured in the data set. The underlying idea is that causation can be distinguished from correlation if several independent causes can be observed. The causal model produces two outputs. First, a graph shows which variables are directly causal of the outcome of interest, which are causal through upstream pathways and which are outside the causal chain. Second, the model can be used to conduct intervention query or 'what-if' analyses. ${ }^{31}$ This is equivalent to conducting a virtual randomised control trial that quantifies the change in the outcome variable that occurs when a specific intervention is made at a variable.

Since a causal BN relies on estimating conditional dependencies between all combinations of included variables, sample size often poses a de facto constraint on the number of variables that can be included. ${ }^{32}$ Through synthetic data simulations, we estimated that only up to 20 variables could be supported by the household survey sample size; thus, we looked to the significance test results in the predictive model to inform inclusion. Caste, which was not a significant predictor in the predictive model but has previously been established as a variable associated with ID, was also included in the causal model. Since caste is correlated with another important variable, religion, we included caste as a compromise input in the causal modelling, to further reduce the number of variables. Income, which was not a significant predictor in the predictive model but has been argued to be an associated variable as well, was not included because there were already two other closely correlated variables commonly used to indicate socioeconomic status (ie, education and electricity). ${ }^{33}$ To improve computational efficiency given our sample size, some variables were recoded from continuous to categorical, or were condensed to include three or fewer categories. ${ }^{32}$ These decisions were consulted on with domain experts to ensure that the cutoffs remained relevant to programme policies. The variables selected are listed in table 1 . 
Table 1 Summary of variables used in predictive model, causal model and segmentation

\begin{tabular}{|c|c|c|c|c|}
\hline Variable & Response options & $\begin{array}{l}\text { Predictive } \\
\text { model }\end{array}$ & $\begin{array}{l}\text { Causal } \\
\text { machine } \\
\text { learning }\end{array}$ & $\begin{array}{l}\text { Segmentation } \\
(\mathrm{S}) \text { and } \\
\text { profiling }(\mathrm{P})\end{array}$ \\
\hline \multicolumn{5}{|l|}{ Demographics } \\
\hline Education & $\begin{array}{l}0-4 \text { years, } 5-9 \text { years, } 10-12 \text { years, } \\
13+\text { years }\end{array}$ & $\mathrm{x}$ & $x$ & $P$ \\
\hline Parity & $1,2,3,4+$ & $x$ & $x$ & S \\
\hline Religion & Hindu versus other & $\mathrm{x}$ & & $P$ \\
\hline Caste & $\mathrm{ST}, \mathrm{SC}, \mathrm{OBC}$, none of these & $\mathrm{x}$ & $x$ & $P$ \\
\hline Income $^{*}$ & Little versus lot & $\mathrm{x}$ & & $P$ \\
\hline Financial insecurity & 2-item composite; 1-5 Likert scale & $x$ & & $P$ \\
\hline Electricity in home & Yes versus no & $x$ & $x$ & $P$ \\
\hline Household type & Nuclear versus joint/other & $x$ & & $P$ \\
\hline \multicolumn{5}{|l|}{ Internal beliefs } \\
\hline Opinion of hospital facilities & 7-item composite; low versus high & $x$ & & $P$ \\
\hline Opinion of hospital services & 6-item composite; low versus high & $x$ & $x$ & $P$ \\
\hline Rank importance of hospital delivery & Important versus unimportant & $x$ & $x$ & $P$ \\
\hline Risk perception of childbirth & Low versus high & $\mathrm{x}$ & & $P$ \\
\hline Worry about delivery problems & Little versus lot & $x$ & & $P$ \\
\hline Perception of hospital safety & Hospital safer versus home safer & $x$ & $x$ & S \\
\hline $\begin{array}{l}\text { Nurse gives injection to make delivery } \\
\text { easier }\end{array}$ & Agree versus disagree & $x$ & & $P$ \\
\hline $\begin{array}{l}\text { Hospital is not necessary if birth } \\
\text { attendant is good }\end{array}$ & Agree versus disagree & $\mathrm{x}$ & & $P$ \\
\hline $\begin{array}{l}\text { Hospital is not necessary if past home } \\
\text { delivery }\end{array}$ & Agree versus disagree & $x$ & & $P$ \\
\hline Pregnant women attract evil spirits & Agree versus disagree & $\mathrm{x}$ & & $P$ \\
\hline False beliefs about ANC checkups & 3-item composite; few versus many & $x$ & & $P$ \\
\hline Barriers to ANC checkups & 5-item composite; few versus many & $x$ & & $P$ \\
\hline Knowledge of IFA & Percent correct recall $(0-100)$ & $x$ & & $P$ \\
\hline Agency & 10-item composite; $1-5$ Likert scale & $x$ & & $P$ \\
\hline Insecurity & 2-item composite; 1-5 Likert scale & $x$ & & $P$ \\
\hline Conscientiousness & 3-item composite; 1-5 Likert scale & $\mathrm{x}$ & & $P$ \\
\hline Empathy & 1-5 Likert scale & $x$ & & $P$ \\
\hline Openness & 2-item composite; 1-5 Likert scale & $\mathrm{x}$ & & $P$ \\
\hline Optimism & 1-5 Likert scale & $x$ & & $P$ \\
\hline Neuroticism & 1-5 Likert scale & $x$ & & $P$ \\
\hline \multicolumn{5}{|l|}{ Structural } \\
\hline Social norms & Low versus high & $\mathrm{x}$ & $x$ & $P$ \\
\hline Hospital distance & $\begin{array}{l}0-20 \text { min versus } 21-40 \text { min versus } \\
40+\text { min }\end{array}$ & $x$ & $x$ & $P$ \\
\hline Labour start time & $\begin{array}{l}\text { Middle of the night versus day versus } \\
\text { evening }\end{array}$ & $\mathrm{x}$ & $x$ & $P$ \\
\hline Money borrowed* & None versus some & $x$ & $x$ & $P$ \\
\hline Incentive awareness & Yes versus no & $x$ & $x$ & $P$ \\
\hline \multicolumn{5}{|l|}{ Influencers } \\
\hline Discussed delivery location with ASHA & True versus false & $X$ & & $P$ \\
\hline
\end{tabular}




\begin{tabular}{|c|c|c|c|c|}
\hline Variable & Response options & $\begin{array}{l}\text { Predictive } \\
\text { model }\end{array}$ & $\begin{array}{l}\text { Causal } \\
\text { machine } \\
\text { learning }\end{array}$ & $\begin{array}{l}\text { Segmentation } \\
\text { (S) and } \\
\text { profiling (P) }\end{array}$ \\
\hline Primary decision maker & $\begin{array}{l}\text { Self versus husband versus mother- } \\
\text { in-law versus other }\end{array}$ & $x$ & $x$ & $\mathrm{P}$ \\
\hline People for social support & Few versus many & $\mathrm{X}$ & & $\mathrm{P}$ \\
\hline Number of ASHA home visits & $\begin{array}{l}\text { None versus } 1-2 \text { versus } 3-4 \text { versus } \\
5+\end{array}$ & $x$ & $x$ & $\mathrm{P}$ \\
\hline \multicolumn{5}{|l|}{ Behaviour } \\
\hline Pregnancy registration & $\begin{array}{l}\text { Not registered versus first trimester } \\
\text { versus second trimester versus third } \\
\text { trimester }\end{array}$ & $x$ & & $\mathrm{P}$ \\
\hline Delivery plan & $\begin{array}{l}\text { Planned ahead of time versus last } \\
\text { minute decision }\end{array}$ & $x$ & $x$ & S \\
\hline Number of ANC checkups & $0-9$ & $X$ & $X$ & $\mathrm{P}$ \\
\hline Take IFA during pregnancy & $\begin{array}{l}\text { None versus less than recommended } \\
\text { amount versus recommended amount } \\
\text { or more }\end{array}$ & $X$ & $\mathrm{X}$ & $\mathrm{P}$ \\
\hline
\end{tabular}

Some variables were constructed from several items in the questionnaire. $X$ indicates variables as inputs.

*Variables that were only assessed for male head of household $(\mathrm{HOH})$; for women who did not have a male $\mathrm{HOH}$ interviewed, a median imputation method was used to generate these estimates. Relevant survey questions are listed in online supplemental information. ANC, antenatal care; ASHA, Accredited Social Health Activists; IFA, iron and folic acid.

To generate our causal BNs we used GNS Healthcare's proprietary Reverse Engineering and Forward Simulation platform, ${ }^{34} 35$ which uses the Markov Chain Monte Carlo algorithm to search for the best causal structure. For all variables, we conducted a series of what-if analyses for the ID outcome. The results are plotted as odds ratios (ORs) for ID. The population attributable fractions $(\mathrm{PAFs})^{36}$ for variables in the causal model were also estimated using what-if analysis. The PAF of a variable from a causal model is interpreted as the proportion of home deliveries that is preventable with an intervention on that variable.

Duplicate entries $(n=650)$, women who reported they had an abortion $(\mathrm{n}=5116)$, women who reported they had a stillbirth $(\mathrm{n}=1176)$, women whose babies did not survive at least 1 day $(n=618)$ and women whose babies at the time of survey were less than 1 day old $(n=388)$ were excluded from the analysis, leaving a final analytic sample of 49840 women (see online supplemental figure 2 for full analytical sample flowchart).

\section{Variance decomposition}

Our data set had two main sources of heterogeneitygeographical variables (ie, districts and blocks), and individuals' characteristics (eg, behaviours and perceptions). We conducted variance decomposition to better understand the relative proportion of variance in delivery location attributable to these two sources before the segmentation analysis. We used mixed-effect logistic regression to fit a null (intercept-only) model, with the geographical units of block and district included as random effects. ${ }^{37}$ This generates the proportion of variance attributed to both the district and block level, with the residual variance representing all other sources of variance contribution, including individual-level factors. For completeness, we also checked the variance attributable to ASHAs in addition to geographical variables.

\section{Segmentation}

To segment women into subgroups, we used a $\chi^{2}$ automatic interaction detection analysis (CHAID) decision tree algorithm on the full set of 41 variables used in the predictive model (table 1). ${ }^{38}$ Decision tree algorithms are particularly well-suited to data in which the target outcome is defined (eg, ID) and are advantageous in their ability to handle categorical and continuous variables simultaneously. They are also easy to understand, visualise and interpret, making them highly actionable.

We employed a top-down pruning approach by sequentially modifying the stopping criteria (ie, minimum number of cases per node, maximum tree depth and alpha threshold) to be more stringent. We used a 10-fold cross-validation method to evaluate generalisation error; the tree with the simplest structure and lowest prediction error and generalisation error was chosen as the final model.

After the final tree was constructed, that final data subset with no further splits defined one segment. Each of these segments was profiled to determine how they differed from each other. All variables in the predictor set were profiled using $\chi^{2}$ and one-way analyses of variance. Additionally, we examined home delivery classification (elective versus non-elective) and reasons for home delivery. In some cases, the continuous version 
of the measure was used to allow for greater sensitivity in detecting differences between segments. The final profiles generated for each segment were based on a combination of the interpretation of practically meaningful differences between segments.

\section{RESULTS}

\section{Most home deliveries are non-elective}

Eighteen per cent of women reported delivering their baby at home. The majority $(59.6 \%, 95 \%$ CI $56.4 \%$ to $62.8 \%$ ) of at-home deliveries were non-elective, $29.8 \%$ (95\% CI $26.8 \%$ to $32.8 \%$ ) were elective and $10.6 \%(95 \%$ CI $9.6 \%$ to $11.6 \%$ ) were classified as 'other'. The most common reason given for non-elective home deliveries was that the baby came too quickly $(40.0 \%, 95 \%$ CI $36.8 \%$ to $43.2 \%$ ); $13.9 \%$ (95\% CI $11.7 \%$ to $16.1 \%$ ) of women also reported delivering at home because labour started in the middle of the night (see online supplemental table 1 for full descriptive statistics). The most common reasons for elective at-home delivery were the perception that it was more convenient $(21.8 \%, 95 \%$ CI $19.0 \%$ to $24.6 \%$ ), followed by a preference for the village birth attendant $(7.0 \%, 95 \%$ CI $5.4 \%$ to $8.6 \%)$.

\section{Hospital delivery is associated with a broad set of factors}

Sixteen variables were significantly associated with ID. In addition to previously established demographic correlates of hospital delivery in India, ${ }^{20}{ }^{39-42}$ we found that beliefs, perceptions and behaviour were key predictors of delivery location. For example, one of the strongest predictors of ID was having a delivery plan. Having a delivery plan means that the woman reported that her household had planned ahead of time to deliver the baby in the place (a particular health facility or at home) where the baby was in fact eventually delivered. Women who said they delivered in their planned location were far more likely to have delivered in a hospital than those who said it was a last-minute decision ( $\mathrm{OR}=4.91,95 \%$ CI 3.84 to 6.28$)$. Women who believed home is safer than the hospital were much less likely to deliver in a hospital $(\mathrm{OR}=0.23,95 \%$ CI 0.18 to 0.31 ) and those who were unaware of ID incentives were less likely to have delivered there $(\mathrm{OR}=0.46$, $95 \%$ CI 0.34 to 0.63 ). While the significant increase in ID thus far has been attributed to financial incentives, which were also found to be a significant predictor $(\mathrm{OR}=2.18$, $95 \%$ CI 1.59 to 2.99$)$, a minority of women $(13.8 \%)$ were not aware of it in our survey. The perception that ID is the social norm in a woman's community increased the odds that she would deliver there $(\mathrm{OR}=1.76,95 \%$ CI 1.38 to 2.25). While previous studies have shown that income, education and proxy for wealth (eg, electricity) are associated with delivery locations, ${ }^{43}$ our analysis showed only the latter two to be significant. We speculate that this is because these factors are very much correlated with each other. ${ }^{44}$ Full results for the regression model are available in online supplemental material.

\section{BN identify causal factors driving ID}

Figure 1 shows the causal BN learnt from the household survey. Several variables are directly causal to (ie, one edge away from) delivery location: having a perception that ID is safer than home, having a predetermined delivery plan, being aware of ID incentives, education level of the mother and being a first-time parent. The number of ASHA home visits plays a key role in promoting incentive awareness and delivery planning.

Perception of ID being a safer option, and further upstream, the amount of ANC checkups, are two important 'gateway' variables that are central to many causal pathways in the network. Perception of ID being a safer option also factors into delivery planning. The number of ANC checkups is particularly interesting: even just 1-2 checkups lead to positive opinions about ID, knowledge and awareness of the health services (and incentives), and committed behaviours (eg, delivery plan), all of which contribute to the location of delivery.

Upstream, the mother's educational level-itself closely related to her caste-and perceiving ID as the social norm appear to be important internal and external causes, respectively, that modulate other downstream behaviours and opinions.

Several variables that were associated with delivery location in the predictive model-such as distance to the nearest hospital and the time of labour onset-were not found to be causal factors. Similarly, the primary decision-maker of delivery location, whether the family borrowed money, and general opinions about hospital services were associated with ID but not causal of it. They appear to be conflated with ID in the predictive model due to some common upstream causes (such as delivery planning). Finally, we observed that a higher number of ANC checkups leads to more ASHA home visits, rather than the other way around.

The what-if analyses (figure 2) showed that having a delivery plan is by far the most influential cause of ID; women had six times the odds of delivering in public facilities if there was a delivery plan than if there was not. Almost just as causal (greater than five times the odds) is perceiving that ID is safe. This result suggests key intervention areas for focusing programme resources. Incentive awareness and mother's education are also important (greater than three times odds), followed by whether the woman is a first-time parent (two times). The number of ANC checkups, home visits and perceptions of ID being a social norm are significant causes with more moderate ORs.

The PAFs show that delivery planning and perceptions of safety are high-value targets (for the full table, see online supplemental material). For delivery planning, $46 \%$ of home delivery can be converted to ID by having a delivery plan. For perceptions of hospital safety, $26 \%$ of home deliveries can be converted by convincing women the hospital is safer. Though awareness of financial incentives is directly causal of delivery location, the PAF is low 


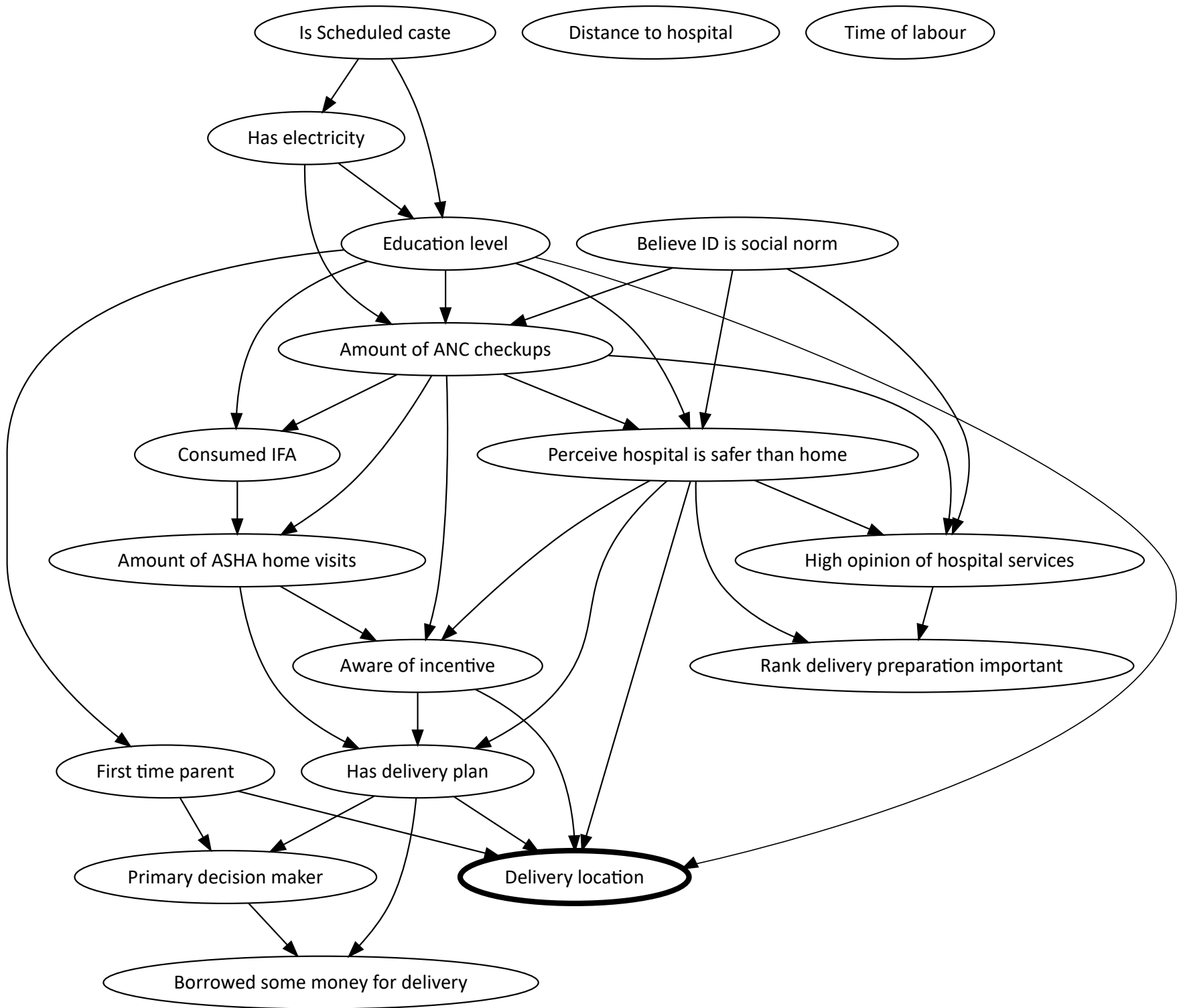

Figure 1 Causal graph depicting causal relationships. ANC, antenatal care; ASHA, Accredited Social Health Activists; ID, institutional delivery; IFA, iron and folic acid.

(15\%) because of the existing high awareness of the incentives.

\section{General conclusions of the causal model of the household data are consistent with the causal model of another independently collected data set}

To confirm our results and explore the role of additional variables, we built another causal model on the CBTS data. We obtained similar results for variables that overlapped with the household survey: having a delivery plan, checkups and education level were all directly causal of public-hospital delivery. Most of the indirect causes for ID found in the household model were also found to be indirectly causal in the CBTS model (online supplemental material). These results suggest that the causal relationships we found are robust in the Uttar Pradesh region.
The CBTS did not include perception and other variables present in the household survey. For example, haemoglobin check in the third trimester was found to be causal in the CBTS, presumably being a proxy of the quality of prenatal checkups. While distance to hospital was not causal in the household model, identification of transportation vehicle was a causal variable in the CBTS model.

\section{Individual heterogeneity matters the most in determining delivery location}

In the variance decomposition analysis, we found that $20 \%$ of the variance in delivery location can be attributed to geography (10\% to the district level, $10 \%$ to the block level). The residual variance-including (potentially) lower geographic and individual-level factorsaccounted for $80 \%$ of the variance in delivery location. 


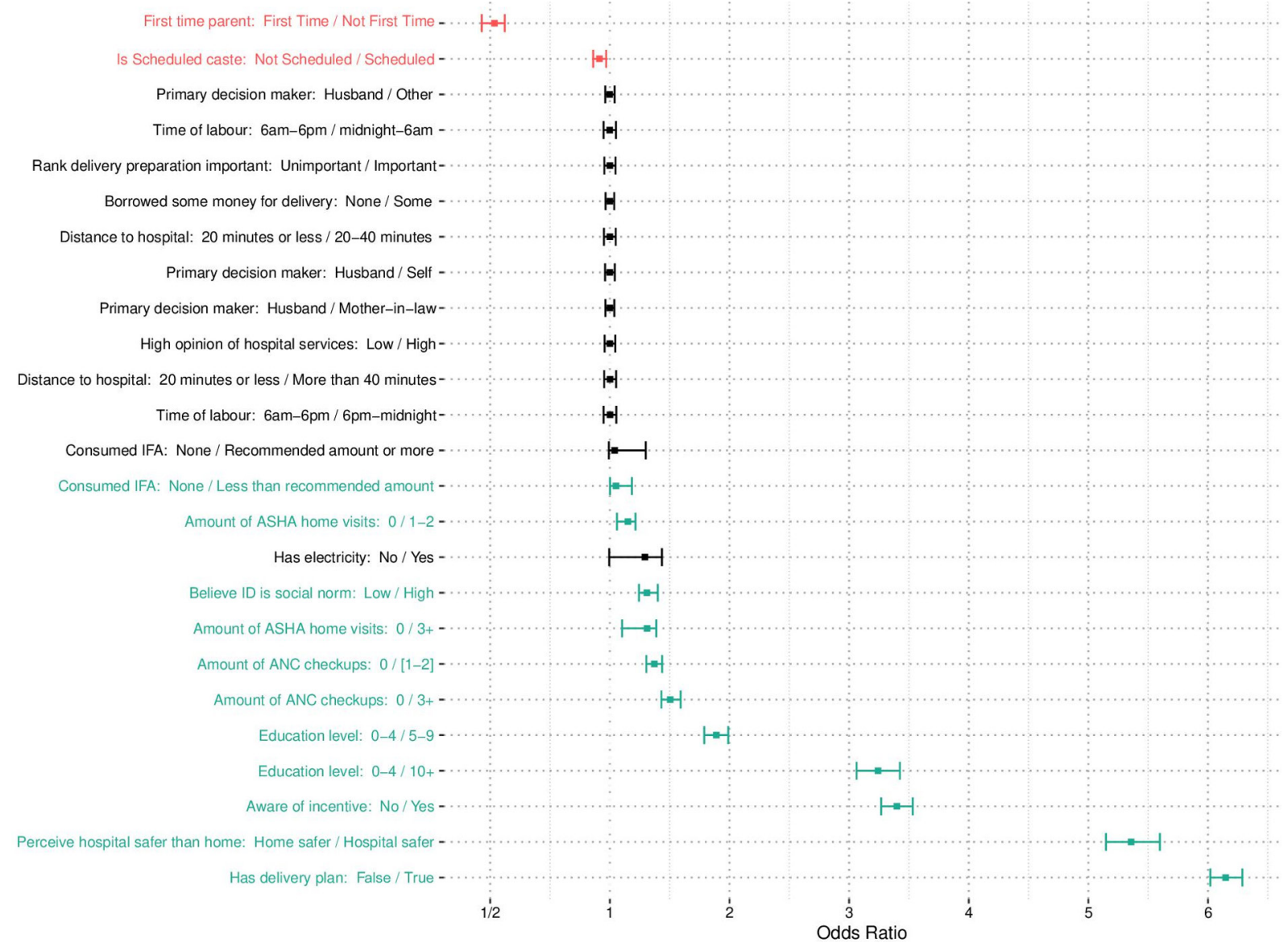

Figure 2 What-if analysis (reference vs intervention); interventional OR of institutional versus home delivery with $95 \%$ Cls on the simulation. ORs significantly greater than 1 (green) indicate higher odds of delivering in a facility; ORs significantly less than 1 (red) indicate higher odds at delivering at home. ANC, antenatal care; ASHA, Accredited Social Health Activists; ID, institutional delivery; IFA, iron and folic acid.

When we reanalysed to include variance attributable to ASHAs, we found that $17 \%$ of the variance in delivery location can be attributed to geographic levels $(8 \%$ to district, $9 \%$ to block) and $14 \%$ to ASHAs, with residual variance accounting for $69 \%$ of the variance in delivery location.

These results indicate that, although geographic features and specific ASHAs do contribute to the likelihood of a mother delivering in a hospital, individual-level factors are far more important in determining delivery location.

\section{Four segments can explain individual-level heterogeneity}

The decision tree analysis segmented women into four groups based on demographics, behaviours and behaviour correlates (figure 3). All branches in the decision tree were made based on a $p$ value of $<0.0001$. The first branch was made on perceptions of hospital safety. Among women who believe the hospital is safer, the next branch was made on delivery planning. Among women who believe the home is safer than the hospital, the tree branched based on parity (first-time births vs two or more past deliveries).

\section{Segment profiling}

The results of the profiling analysis, including the significance testing of all profiled variables, are presented in table 2. Here, we highlight a few key variables that are not predictive of the target outcome, but differentiate the segments and so could be used to identify segment members for targeted interventions.

Segment 1 are the disempowered first-timers. These are women giving birth for the first time who believe the home is safer than the hospital. They tend to be younger, have more years of education, and fewer financial barriers (ie, indicators of poverty). They tend to live in joint family households and report more often than women in the other segments that their mother-in-law is responsible for making the decision about where they deliver. They have some false beliefs about pregnancy and delivery. These women do not perceive ID to be the social norm in their community. They account for $7 \%$ of all home deliveries.

Segment 2 are the traditional home deliverers. They are experienced women who believe the home is safer for delivery. They tend to be older, less educated and have many financial barriers. They tend to live in nuclear family households, and are more likely to decide 


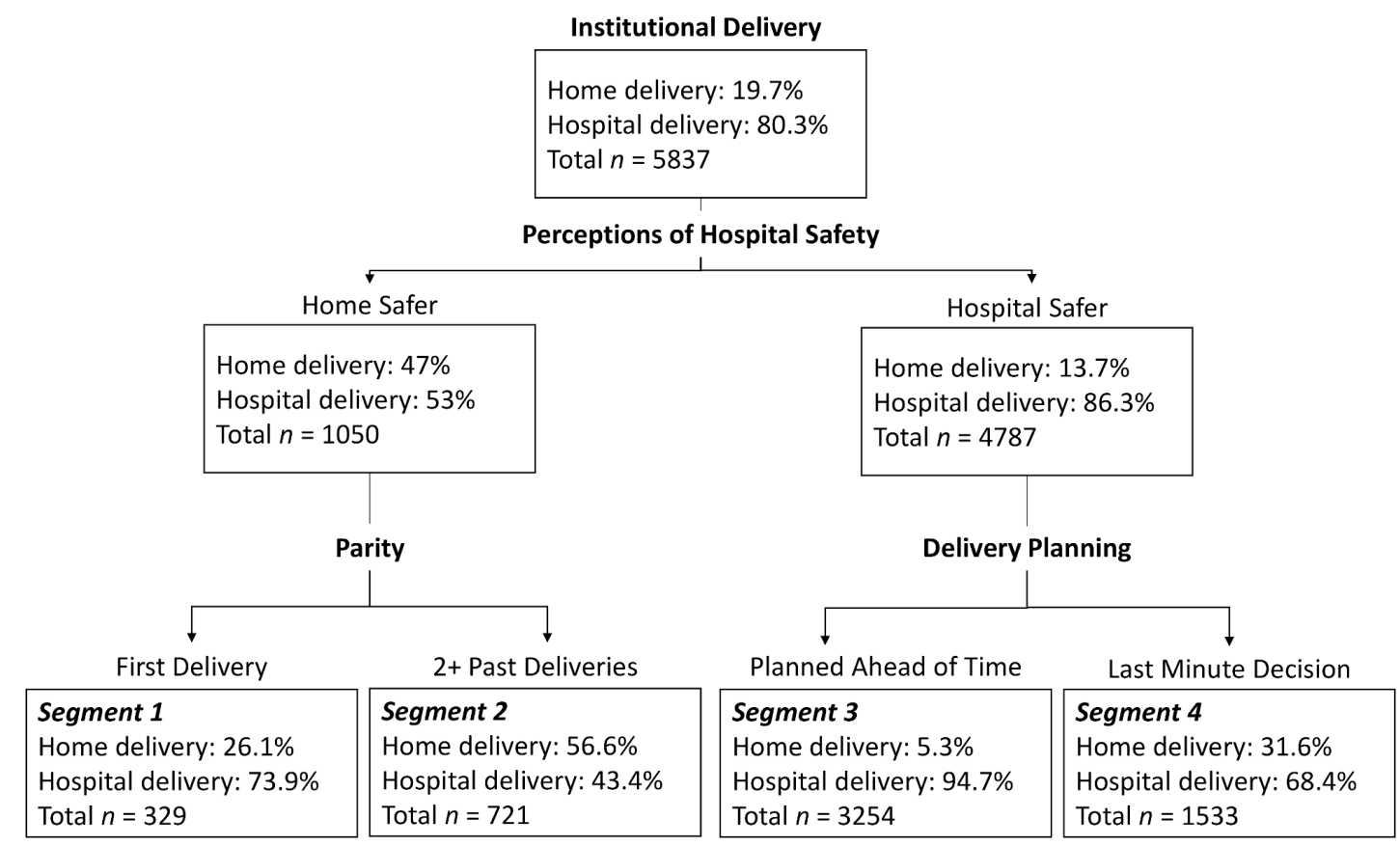

Figure 3 Final decision tree; values within nodes indicate the proportion of each subgroup delivering at home or at a hospital facility and the total sample represented within the node. The model correctly classified $81.9 \%$ of cases; sensitivity (ie, correct classification of institutional delivery) was $93.3 \%$ and specificity (ie, correct classification of home delivery) was $35.4 \%$. The generalisation risk estimate was .181 ( $\mathrm{SE}=0.005)$, indicating the model performed comparably on the validation samples.

Values are based on the cross-validated model.

themselves where to deliver. They have many false beliefs about pregnancy and delivery, and poor and infrequent contact with the ASHA. These women do not perceive ID to be the social norm in their community. They account for $35 \%$ of all home deliveries.

Segment 3 are the hospital seekers. They believe the hospital is safer for delivery and plan ahead to deliver there. They are more educated, have fewer financial barriers to ID and are aware of the ID incentive. They have low rates of false beliefs about pregnancy and delivery. These women believe that ID is the social norm in their community. They have good ANC practices, frequent and positive contact with the ASHA, and report a high level of social support. They account for $15 \%$ of all home deliveries.

Segment 4 are the informed poor planners. These women believe the hospital is safer but lack a delivery plan. In terms of education and financial barriers they are better off than segment 2 but worse off than segments 1 and 3 . They tend to have positive perceptions of hospitals and fewer false beliefs. They perceive ID to be the social norm in their community. These women have less frequent and positive contact with the ASHA. They account for $42 \%$ of all home deliveries across the four segments.

We also examined the subset of home deliveries within each segment and found that home delivery risk is well differentiated. Seventy-seven per cent of all home deliveries occur just in segments 2 and 4, but for very different reasons between segments. The home deliveries that occurred among the informed poor planners (segment
4) were almost entirely non-elective $(83.6 \%)$, the most common reason given being that the baby came too quickly $(54.9 \%)$. In contrast, more than half $(56.2 \%)$ of all elective home deliveries are accounted for by traditional home deliverers (segment 2), who most commonly reported that it was more convenient $(36.3 \%)$.

\section{DISCUSSION}

By applying a PxPH approach, using novel data and integrating multiple ML methodologies, we were able to generate more nuanced insights into why $18 \%$ of women in Uttar Pradesh continue to deliver at home. Insights from any single method would have provided only a piece of the picture, potentially misdirecting intervention strategies.

First, by focusing on collecting the why data, ${ }^{27}$ we enabled findings that ran counter to some of the conventional wisdom. Respondents reported that most home deliveries were not a matter of preference, but occurred for non-elective reasons. Factors outside of a mother's perceived control-for example, labour progressing too quickly to get to the hospital in time-are associated with home delivery. Those who delivered at home because it was the preferred location accounted for a much smaller subset of women. Had the data not included this critical aspect, we would have missed highly associative factors to be included in subsequent analyses.

We used traditional methods to construct a predictive model of ID using the broad set of drivers and barriers; this analysis confirmed past research showing, 
Table 2 Decision tree profiling

\begin{tabular}{|c|c|c|c|c|}
\hline Variable & $\begin{array}{l}\text { Segment 1: } \\
\text { disempowered } \\
\text { first-timers }\end{array}$ & $\begin{array}{l}\text { Segment 2: } \\
\text { traditional home } \\
\text { deliverers }\end{array}$ & $\begin{array}{l}\text { Segment 3: } \\
\text { hospital } \\
\text { seekers }\end{array}$ & $\begin{array}{l}\text { Segment 4: } \\
\text { informed poor } \\
\text { planners }\end{array}$ \\
\hline \multicolumn{5}{|l|}{ Variables in segmentation } \\
\hline Believes home is safer (\%) & $100_{a}$ & $100_{a}$ & $0_{b}$ & $0_{b}$ \\
\hline Has a delivery plan (\%) & $52.9 \mathrm{a}$ & $57_{\mathrm{a}}$ & $100_{b}$ & $0_{c}$ \\
\hline Average parity & $1.00(0.00)_{a}$ & $3.44(1.67)_{b}$ & $2.43(1.59)_{c}$ & $2.46(1.68)_{c}$ \\
\hline \multicolumn{5}{|l|}{ Demographics } \\
\hline Years of education & $6.24(5.40)_{a, c}$ & $3.06(4.56)_{b}$ & $6.07(5.43)_{c}$ & $5.72(5.43)_{a}$ \\
\hline Age & $22.19(2.68)_{a}$ & $26.66(4.16)_{b}$ & $25.12(3.85)_{c}$ & $25.16(4.00)_{c}$ \\
\hline Hindu religion (\%) & $85.4_{a, b}$ & $73.5_{\mathrm{c}}$ & $86_{b}$ & $82.5_{a}$ \\
\hline Muslim religion (\%) & $14.3_{\mathrm{a}, \mathrm{b}}$ & $26.2_{c}$ & $13.9_{b}$ & $17.1_{\mathrm{a}}$ \\
\hline Scheduled tribe (\%) & $4.9_{a}$ & $4.0_{a}$ & $3.4 a$ & $4.4_{a}$ \\
\hline Scheduled caste (\%) & $27.4_{a}$ & $29.0_{a}$ & $29.2_{a}$ & $27.3_{a}$ \\
\hline Other backward class (\%) & $55.6_{a}$ & $57.6_{a}$ & $55.6_{a}$ & $56.1_{a}$ \\
\hline Upper caste $(\%)$ & $12.2_{a}$ & $9.4 \mathrm{a}$ & $11.7_{\mathrm{a}}$ & $12.2_{a}$ \\
\hline Low income (\%) & $70.2_{a}$ & $76.6_{b}$ & $75.3_{b}$ & $74.7_{\mathrm{a}, \mathrm{b}}$ \\
\hline No electricity in home (\%) & $21.3_{a, b}$ & $29.3_{c}$ & $21.5_{b}$ & 24.6 \\
\hline Financial insecurity & $2.76(1.10)_{a}$ & $3.12(1.00)_{b}$ & $2.99(1.05)_{c}$ & $3.01(1.06)_{c}$ \\
\hline Joint family household (\%) & $799_{a}$ & $51_{b}$ & $67.5_{c}$ & $63_{d}$ \\
\hline \multicolumn{5}{|l|}{ Internal/beliefs } \\
\hline High opinion of hospital facilities (\%) & $37.7_{\mathrm{a}}$ & $35.1_{a}$ & $46.3_{b}$ & $46.8_{b}$ \\
\hline High opinion of hospital services (\%) & $39.2_{a}$ & $42.9_{a}$ & $54.9_{\mathrm{b}}$ & $55.3_{b}$ \\
\hline Ranks hospital delivery as important (\%) & $30.4_{a}$ & $33.8_{a, b}$ & $39.8_{c}$ & $38.1_{b, c}$ \\
\hline High risk perception of childbirth (\%) & $63.8_{a, b}$ & $63.7_{b}$ & $67.7_{\mathrm{a}}$ & $66.4_{\mathrm{a}, \mathrm{b}}$ \\
\hline High worry about delivery problems (\%) & $62.3_{a, b}$ & $66.9_{\mathrm{b}}$ & $62.3_{\mathrm{a}}$ & $61.5 \mathrm{a}$ \\
\hline $\begin{array}{l}\text { Believes nurse can give injections to } \\
\text { make delivery easier (\%) }\end{array}$ & $75.1_{a}$ & $66.4_{b}$ & $72.2_{a}$ & $74.5_{a}$ \\
\hline $\begin{array}{l}\text { Believes hospital is not necessary if there } \\
\text { is a skilled Dai (\%) }\end{array}$ & $68.1_{a}$ & $69.3_{a}$ & $35.6_{b}$ & $41.4_{c}$ \\
\hline $\begin{array}{l}\text { Believes hospital is not necessary if } \\
\text { delivered at home in the past }(\%)\end{array}$ & $67.2_{a}$ & $68.5_{a}$ & $38.9_{b}$ & $40.5_{b}$ \\
\hline $\begin{array}{l}\text { Believes pregnant women going out } \\
\text { attract evil spirits (\%) }\end{array}$ & $60.8_{\mathrm{a}, \mathrm{b}}$ & $65.9_{b}$ & $59.4_{a}$ & $60.3_{a}$ \\
\hline False beliefs about ANC checkups (\%) & $51.7_{\mathrm{a}}$ & $54.4_{a}$ & $43.5_{b}$ & $42.8_{b}$ \\
\hline High opinion of ANC checkups (\%) & $43.8 \mathrm{a}$ & $40.5 \mathrm{a}$ & $50_{b}$ & $51_{b}$ \\
\hline Agency & $2.93(0.47)_{a}$ & $2.95(0.46)_{a}$ & $3.01(0.47)_{b}$ & $2.99(0.48)_{b}$ \\
\hline Insecurity & $3.74(0.94)_{a}$ & $3.78(0.84)_{a}$ & $3.81(0.90)_{a}$ & $3.88(0.87)_{b}$ \\
\hline Conscientiousness & $3.63(0.64)_{a, b}$ & $3.56(0.64)_{b}$ & $3.69(0.62)_{a}$ & $3.69(0.63)_{a}$ \\
\hline Empathy & $3.36(1.24)_{a, b}$ & $3.23(1.20)_{a}$ & $3.36(1.16)_{b}$ & $3.33(1.16)_{a, b}$ \\
\hline Openness & $3.99(0.85)_{a}$ & $3.76(0.91)_{b}$ & $3.99(0.85)_{a}$ & $3.97(0.83)_{a}$ \\
\hline Optimism & $3.80(1.00)_{a, b}$ & $3.75(1.01)_{b}$ & $3.85(1.00)_{a}$ & $3.88(0.96)_{a}$ \\
\hline Neuroticism & $3.51(1.15)_{\mathrm{a}}$ & $3.56(1.15)_{a}$ & $3.53(1.12)_{a}$ & $3.45(1.12)_{a}$ \\
\hline \multicolumn{5}{|l|}{ Structural } \\
\hline Perceives ID as social norm (\%) & $47.7_{\mathrm{a}}$ & $45.1_{\mathrm{a}}$ & $69.5_{b}$ & $63.5_{c}$ \\
\hline Lives 40+ min from hospital (\%) & 15.9 a & $18.6{ }_{a}$ & $19.1_{\mathrm{a}}$ & $19.8 \mathrm{a}$ \\
\hline $\begin{array}{l}\text { Labour starts in the middle of the night } \\
\text { (\%) }\end{array}$ & $24.8_{\mathrm{a}, \mathrm{b}}$ & $29_{b}$ & $25.4_{a}$ & $25.4_{\mathrm{a}, \mathrm{b}}$ \\
\hline
\end{tabular}


Table 2 Continued

\begin{tabular}{|c|c|c|c|c|}
\hline Variable & $\begin{array}{l}\text { Segment 1: } \\
\text { disempowered } \\
\text { first-timers }\end{array}$ & $\begin{array}{l}\text { Segment 2: } \\
\text { traditional home } \\
\text { deliverers }\end{array}$ & $\begin{array}{l}\text { Segment 3: } \\
\text { hospital } \\
\text { seekers }\end{array}$ & $\begin{array}{l}\text { Segment 4: } \\
\text { informed poor } \\
\text { planners }\end{array}$ \\
\hline Borrows no money for delivery (\%) & $76_{a}$ & $81.7_{b, c}$ & $84_{c}$ & $79.8_{a, b}$ \\
\hline Aware of ID incentive (\%) & $80.9_{a}$ & $79.8_{a}$ & $89.8_{b}$ & $82.8_{a}$ \\
\hline \multicolumn{5}{|l|}{ Influencers } \\
\hline $\begin{array}{l}\text { Discusses delivery location with ASHA } \\
(\%)\end{array}$ & $30.7_{\mathrm{a}}$ & $23.4_{b}$ & $42.5_{c}$ & 32 \\
\hline Mother is primary decision maker (\%) & $25.9_{a, b, c}$ & $31.6_{c}$ & $26.8_{b}$ & $21.3_{a}$ \\
\hline $\begin{array}{l}\text { Mother-in-Law is primary decision maker } \\
(\%)\end{array}$ & $18.3_{a}$ & $11.2_{b}$ & $15.7_{\mathrm{a}, \mathrm{c}}$ & $13.7_{b, c}$ \\
\hline High social support (\%) & $61.4 \mathrm{a}$ & $65.6_{a, b}$ & $72.5_{c}$ & $67.7_{b}$ \\
\hline Number of ASHA visits & $3.49(2.80)_{a, c}$ & $3.15(2.75)_{a}$ & $4.02(2.65)_{b}$ & $3.29(2.64)_{c}$ \\
\hline \multicolumn{5}{|l|}{ Behaviour } \\
\hline Did not take any IFA (\%) & $23.1_{a}$ & $31.8_{b}$ & $14_{c}$ & $20.8_{a}$ \\
\hline Took recommended amount of IFA (\%) & $16.1_{a}$ & $9.8_{b}$ & 16 & $11.4_{b}$ \\
\hline Number of ANC checkups & $2.23(1.76)_{a}$ & $1.73(1.52)_{b}$ & $2.58(1.70)_{\mathrm{C}}$ & $2.39(1.70)_{a}$ \\
\hline Pregnancy registered in first trimester (\%) & $53.8_{a}$ & $45.2_{b}$ & $59.8_{c}$ & $54.4_{a}$ \\
\hline
\end{tabular}

Values with the same subscript in the same row do not significantly differ. Values with different subscripts in the same row are significantly different, $p<0.05$.

ANC, antenatal care; ASHA, Accredited Social Health Activists; ID, institutional delivery; IFA, iron and folic acid.

for example, that higher education, lower parity, more ANC checkups and contact with the ASHA all are associated with increased odds of hospital delivery. ${ }^{45}{ }^{46}$ We also found that perceptual factors are correlated with delivery location. For example, one of the strongest predictors of ID was the perception of safety. Additionally, we found that the normative behaviour of a woman's community was strongly associated with ID. These findings are consistent with previous research in Uttar Pradesh done on a smaller scale and relying on qualitative interviews. ${ }^{20}$

The results of the causal ML analysis confirmed that perceptions of hospital safety and delivery planning had a direct causal effect on delivery location. Additionally, several variables shown to be associated with ID in the predictive model were ruled out as causal. For example, we saw that distance to the nearest hospital-a structural factor associated with ID in our predictive model-was not causally linked to location of delivery. Similarly, while respondents indicated that the time of onset of labour was an important reason for home delivery, it was not causal in the causal model. Although other research has shown that distance is associated with ID, ${ }^{4147} 48$ the lack of causal influence in our study may be due to the success of the financial incentive programme and other initiatives specifically designed to reduce this barrier to ID. ${ }^{49}$ In the CBTS causal model, we found that identifying a vehicle for hospital transport was causally related to ID. While we cannot directly compare the two models, this suggests that the ability to access a healthcare facility, rather than absolute distance or the labour onset time, may be important in getting women to deliver there. ${ }^{41}$
In contrast to other research, ${ }^{50}$ we found that the primary decision-maker of delivery location was not a direct cause of delivery location. In other words, changing the decision-maker does not influence whether a mother delivers at home or in the hospital. However, in other work we show that ASHAs were more effective when counselling husbands (who are most often the primary decision-maker) than when they counsel women, suggesting that the ASHA is a more effective channel for promoting behaviour change than the specific household dynamics. ${ }^{51}$

Together, these analyses point to several key areas for intervention efforts. Since resource constraints and other factors may not make it practical to design fully precision interventions customised to each individual, we need instead to exploit any underlying heterogeneity in the population to target distinct groups of women. ${ }^{52}$ The results of our variance decomposition analysis indicate that although some variation in ID is attributable to geographic levels (district and block) or the ASHA, most of the variance $(69 \%)$ is attributable to individuallevel differences. It is possible that heterogeneity exists at a lower geographic level-for example, between ASHA areas or villages. However, we were unable to examine this due to sample size constraints. We used an ML algorithm to segment the sample population of women into four types with just three predictors-perceptions of safety, delivery planning and parity.

We then profiled these segments across several other variables to understand, descriptively, how they differed and to identify possible channels for interventions. For 
example, a key differentiator of the informed poor planners segment is that they did not have a delivery plan; based on the predictive and causal model, promoting delivery planning is likely to be a high-value intervention target, and better leveraging the ASHA could be the channel through which to deliver this intervention to these women. In contrast, traditional home deliverers are more likely to be persuaded to deliver in a facility by targeting false beliefs (eg, the perception that the home is safer for delivery than the hospital).

These findings have actionable implications for current intervention design. In Uttar Pradesh, many interventions to encourage women to deliver in hospitals have targeted specific factors (ie, financial barriers) that were assumed to be the same for everyone. This programme was successful in increasing ID for most women, but not all responded in the hoped-for way. Our analysis helps to explain why, and offers a viable path forward for developing and deploying targeted interventions. These should focus on a few key areas-improving delivery planning, behaviour change communications and counselling around hospital and home safety, and transport accessibility. Using public health principles, another potential intervention could be to equip ASHAs with tools to understand which segment a mother falls into and train her to provide targeted support. For some women, this could mean counselling the mother and mother-in-law about ID and emphasising the risk of delivery at home. For others, it could be helping develop a delivery plan and identifying transport to the hospital well in advance of her due date.

We have shown that predictive models, causal models, variance decomposition and decision trees are complementary tools. Traditional methods cannot identify causal relationships in the absence of long, expensive randomised control trials, but a predictive model is useful to narrow down the variables of interest to enable causal ML. However, our study has several limitations. The data requirements for causal ML are more stringent than for predictive models, including the need for a much bigger sample size to produce reliable estimates. ${ }^{32}$ To be computationally feasible, the data are often converted to categorical rather than gradual numerical values. ${ }^{53}$ Non-causal methods trade explanatory power for more relaxed restrictions. While BN excels in modelling observed confounding, in limited circumstances the true causal direction of connected variables may be challenging to recover if the training data lacks variables that are causal to the variables we are interested in (ie, latent common confounder).$^{54}$ It is therefore crucial that prior to causal modelling, domain experts and modellers make sure that all relevant variables are included in the training data, as far as possible. This 'causal sufficiency' requirement is an area of active research in causal discovery. Many traditional statistical models such as regression have accepted performance (goodness of fit) measures. Our causal ML performance is inferred using synthetic datasets with characteristics similar to the empirical input that is modelled. ${ }^{32}$ However, this evaluation step is itself innovative and not typically done in causal ML.

Decision tree algorithms also have limitations. One of the key disadvantages is instability across training samples. ${ }^{55}$ Given that CHAID is based on correlation rather than causation, there is no theoretical basis on which the resultant segments should respond to interventions. Here we were able to verify whether segmenting predictors were also causal by using a causal analysis, which revealed that they indeed were. In future work, we plan to use different methods for incorporating causal and classification algorithms, such as recursive partitioning for heterogeneous causal effects. ${ }^{56}$ Finally, although we can offer recommendations based on our insights, the real test will be when an actual intervention is implemented on the ground and its long-term outcomes evaluated.

\section{CONCLUSION}

Many current studies focus on single methods and singular study outcomes, and it is often difficult to piece together a holistic picture to design field programmes in a more efficient and systematic way. Using ID in Uttar Pradesh as a use case, we have demonstrated how integrating multiple traditional and ML methodologies can inform a PxPH approach. By collecting better data and employing smarter analytic methods, we developed a holistic picture of why $18 \%$ of women continue to deliver at home. This approach can be applied to myriad global health problems, allowing programmes to leverage limited resources most efficiently to tailor interventions.

\section{Author affiliations}

${ }^{1}$ Surgo Foundation, Washington, DC, USA

${ }^{2}$ Centre for Global Public Health, Department of Community Health Sciences, University of Manitoba, Winnipeg, Manitoba, Canada

${ }^{3}$ India Health Action Trust, New Delhi, India

${ }^{4}$ India Health Action Trust, Lucknow, India

${ }^{5}$ National Health Mission, Government of Uttar Pradesh, Lucknow, India

${ }^{6}$ Department of Global Health, University of Washington, Seattle, WA, USA

${ }^{7}$ Department of Global Health and Population, Harvard University T.H. Chan School of Public Health, Boston, Massachusetts, USA

Acknowledgements We thank Yael Caplan and James Baer for their input on the manuscript.

Contributors VSH, KM, MJ and SKS planned, conducted, analysed and reported the work described in the article. HK, BMR, JB, SI, BS, VG, VN and PK planned and conducted the data required for the work.

Funding The authors have not declared a specific grant for this research from any funding agency in the public, commercial or not-for-profit sectors.

Competing interests None declared.

Patient and public involvement Patients and/or the public were not involved in the design, or conduct, or reporting, or dissemination plans of this research.

Patient consent for publication Not required.

Ethics approval The study was approved by Sigma Institutional Review Board (approval number 10032/RB/D/16-17, New Delhi, India). Analysis of the data 
was approved by University of Manitoba Health Research Ethics Board (HS18479, HS19849 and HS20187). All subjects included in the analyses gave consent.

Provenance and peer review Not commissioned; externally peer reviewed

Data availability statement Data are available upon request. Data are available upon request and may require the approval from the Government of Uttar Pradesh, India.

Open access This is an open access article distributed in accordance with the Creative Commons Attribution Non Commercial (CC BY-NC 4.0) license, which permits others to distribute, remix, adapt, build upon this work non-commercially, and license their derivative works on different terms, provided the original work is properly cited, appropriate credit is given, any changes made indicated, and the use is non-commercial. See: http://creativecommons.org/licenses/by-nc/4.0/.

\section{ORCID iD}

Sema K Sgaier http://orcid.org/0000-0002-8311-2686

\section{REFERENCES}

1 Desmond-Hellmann S. Progress lies in precision. Science 2016;353:731-31.

2 Khoury MJ, lademarco MF, Riley WT. Precision public health for the era of precision medicine. Am J Prev Med 2016:50:398-401.

3 Chowkwanyun M, Bayer R, Galea S. "Precision" public health between novelty and hype. N Engl J Med 2018;379:1398-400.

4 Tatem AJ, Huang Z, Narib C, et al. Integrating rapid risk mapping and mobile phone call record data for strategic malaria elimination planning. Malar J 2014;13:1-36.

5 Sgaier SK, Eletskaya M, Engl E, et al. A case study for a psychographic-behavioral segmentation approach for targeted demand generation in voluntary medical male circumcision. Elife 2017;6:e25923.

6 Flaxman AD, Vos T. Machine learning in population health: opportunities and threats. PLoS Med 2018;15:e1002702.

7 Dolley S. Big data's role in precision public health. Front Public Health 2018;6:68.

8 Mooney SJ, Pejaver V. Big data in public health: terminology, machine learning, and privacy. Annu Rev Public Health 2018;39:95-112.

9 Piot P. Machine learning can fix how we manage health on a globa scale, 2019. Available: https://www.wired.co.uk/article/machinelearning-healthcare-ebola [Accessed 4 Jan 2019].

10 Wahl B, Cossy-Gantner A, Germann S, et al. Artificial intelligence (Al) and global health: how can Al contribute to health in resource-poor settings? BMJ Glob Health 2018;3:e000798.

11 Bishop CM. Pattern recognition and machine learning. New York: Springer, 2006

12 Russell S, Norvig P. Artificial intelligence: a modern approach. Malaysia: Pearson, 2016.

13 Alegana VA, Atkinson PM, Lourenço C, et al. Advances in mapping malaria for elimination: fine resolution modelling of Plasmodium falciparum incidence. Sci Rep 2016;6:29628.

14 Dwyer-Lindgren L, Cork MA, Sligar A, et al. Mapping HIV prevalence in sub-Saharan Africa between 2000 and 2017. Nature 2019;570:189-93

15 Golding N, Burstein R, Longbottom J, et al. Mapping under-5 and neonatal mortality in Africa, 2000-15: a baseline analysis for the sustainable development goals. Lancet 2017;390:2171-82.

16 Mutheneni SR, Mopuri R, Naish S, et al. Spatial distribution and cluster analysis of dengue using self organizing maps in Andhra Pradesh, India, 2011-2013. Parasite Epidemiol Control 2018;3:52-61.

17 Sgaier SK, Reed JB, Sundaram M, et al. Interventions to drive uptake of voluntary medical male Circumcision-A collection of impact evaluation evidence. J Acquir Immune Defic Syndr 2016;72(Suppl 4):S257-61.

18 Office of the Registrar General of India (ORGI). Special Bulletin on maternal mortality in India 2014-16. India: New Delhi Office of the Registrar General, 2018.

19 Office of the Registrar General of India (ORGI). Special Bulletin sample registration system. India: New Delhi Office of the Registrar General, 2019.

20 Varma DS, Khan ME, Hazra A. Increasing institutional delivery and access to emergency obstetric care services in rural Uttar Pradesh. J Fam Welfare 2010;56:23-30.

21 Ministry of Health and Family Welfare. Janani Suraksha Yojana: revised guidelines for implementation. New Delhi: Government of India, 2006.
22 Centre for Operations Research and Training (CORT). Assessment of Janani Suraksha Yojana in Uttar Pradesh. Vadodara, 2007.

23 Ministry of Health and Family Welfare. Janani Suraksha Yojana features and frequently asked questions. New Delhi: Government of India, 2006.

24 Joe W, Perkins JM, Kumar S, et al. Institutional delivery in India, 2004-14: unravelling the equity-enhancing contributions of the public sector. Health Policy Plan 2018;33:645-53.

25 Engl E, Sgaier S. Smarter micro-targeting to improve global health outcomes: scaling cluster segmentation on novel types of data for precision public health. 32nd conference on neural information processing systems (NIPS 2018), Montréal, Canada, 2018.

26 Kumar G, Choudhary TS, Srivastava A, et al. Utilisation, equity and determinants of full antenatal care in India: analysis from the National family health survey 4. BMC Pregnancy Childbirth 2019;19:327.

27 Engl E, Sgaier SK. Cubes: a practical toolkit to measure enablers and barriers to behavior for effective intervention design. Gates Open Res 2019;3:886.

28 Uttar Pradesh Technical Support Unit. Community behaviour tracking survey: results of the first round. Government of Utta Pradesh, India, 2015

29 Greenland S, Pearl J, Robins JM. Causal diagrams for epidemiologic research. Epidemiology 1999;10:37-48.

30 Pearl J. Causal diagrams for empirical research. Biometrika 1995;82:669-88.

31 Arora P, Boyne D, Slater JJ, et al. Bayesian networks for risk prediction using real-world data: a tool for precision medicine. Value Health 2019;22:439-45.

32 Butcher B, Huang V, Reffin J. Causal datasheet: an approximate guide to practically assess Bayesian networks in the real world. In: Thorsten Joachims NK, Swaminathan A, Santacatterina M, et al, eds. Conference on neural information processing systems (NIPS 2019). Vancouver, Canada, 2019.

33 Kattula D, Venugopal S, Velusamy V, et al. Measuring poverty in southern India: a comparison of socio-economic scales evaluated against childhood stunting. PLoS One 2016;11:e0160706.

34 Gendelman R, Xing H, Mirzoeva OK, et al. Bayesian network inference modeling identifies Trib1 as a novel regulator of cellcycle progression and survival in cancer cells. Cancer Res 2017:77:1575-85.

35 Xing H, McDonagh PD, Bienkowska J, et al. Causal modeling using network ensemble simulations of genetic and gene expression data predicts genes involved in rheumatoid arthritis. PLoS Comput Biol 2011;7:e1001105.

36 Mansournia MA, Altman DG. Population attributable fraction. BMJ 2018;360:k757.

37 Nakagawa S, Schielzeth $\mathrm{H}$. A general and simple method for obtaining $R^{2}$ from generalized linear mixed-effects models. Methods Ecol Evol 2013;4:133-42.

38 Kass GV. An exploratory technique for investigating large quantities of categorical data. App/ Stat 1980;29:119.

39 Ladusingh L, Holendro Singh C. Rich-Poor gap in maternal care: the case of Northeast India. Asian Population Stud 2007;3:79-94.

40 Mohanty SK, Pathak PK. Rich-poor gap in utilization of reproductive and child health services in India, 1992-2005. J Biosoc Sci 2009;41:381-98.

41 Kesterton AJ, Cleland J, Sloggett A, et al. Institutional delivery in rural India: the relative importance of accessibility and economic status. BMC Pregnancy Childbirth 2010;10:30-8.

42 Dey A, Hay K, Afroz B, et al. Understanding intersections of social determinants of maternal healthcare utilization in Uttar Pradesh, India. PLoS One 2018;13:e0204810.

43 Sahoo J, Singh SV, Gupta VK, et al. Do socio-demographic factors still predict the choice of place of delivery: a cross-sectional study in rural North India. J Epidemiol Glob Health 2015;5:S27-34.

44 Singh-Manoux A, Dugravot A, Smith GD, et al. Adult education and child mortality in India: the influence of caste, household wealth, and urbanization. Epidemiology 2008;19:294-301.

45 Thind A, Mohani A, Banerjee K, et al. Where to deliver? Analysis of choice of delivery location from a national survey in India. BMC Public Health 2008;8:29-36.

46 Seth A, Tomar S, Singh K, et al. Differential effects of community health worker visits across social and economic groups in Uttar Pradesh, India: a link between social inequities and health disparities. Int J Equity Health 2017;16:1-9.

47 Kumar S, Dansereau EA, Murray CJL. Does distance matter for institutional delivery in rural India? Appl Econ 2014:46:4091-103.

48 Sridharan S, Dey A, Seth A, et al. Towards an understanding of the multilevel factors associated with maternal health care utilization in Uttar Pradesh, India. Glob Health Action 2017;10:1287493. 
49 Randive B, Diwan V, De Costa A. India's conditional cash transfer programme (the JSY) to promote institutional birth: is there an association between institutional birth proportion and materna mortality? PLoS One 2013;8:e67452-8.

50 International Institute for Population Sciences. Perceived barriers to utilization of maternal health and child health services: qualitative insights from rural Uttar Pradesh, India. New Delhi, 2010.

51 Smittenaar P BMR, Jain M, Blanchard J, et al. Improving precision of community health worker efforts to drive maternal and newborn health - findings and policy implications from linked cross-sectional data 2020. Under Review.
52 Sgaier SK, Engl E, Kretschmer S. Time to scale Psycho-behavioral segmentation in global development. Stanford Social Innovation Review 2018;16:48-55.

53 Heckerman D. A tutorial on learning with Bayesian networks. Berlin Heidelberg: Springer Berlin Heidelberg, 2008.

54 Pearl J. Causality: models, Reasoning, and inference. New York, Cambridge, U.K: Cambridge University Press, 2000.

55 Kotsiantis SB. Decision trees: a recent overview. Artif Intell Rev 2013;39:261-83.

56 Athey S, Imbens G. Recursive partitioning for heterogeneous causal effects. Proc Natl Acad Sci U S A 2016;113:7353-60. 